\title{
1. Introduction to organizational project management
}

\section{WHAT IS ORGANIZATIONAL PROJECT MANAGEMENT?}

Doing projects in organizations requires careful selection of the 'right' projects and thereafter careful coordination with other projects being carried out by the organization. Numerous issues could be resolved through this coordination in order to avoid clashes between projects. This is often achieved through measures such as the prioritization of projects to assign resources to work on the projects, harmonizing the ways to execute the projects to leverage established working practices, or standardizing the ways project management is governed in order for project managers to know what they are expected to do and how they are held accountable for their responsibilities.

Organizational project management (OPM) addresses this coordination from a broad perspective. Briefly, OPM is the integration of the primarily project (management)-related activities of an organization into a cohesive network of activities which, by themselves and in their interactions, can be understood, planned and managed for the benefit of the organization and its stakeholders. This includes the integration of activities at various layers of an organization's hierarchy or network, such as strategic decisions (e.g. management's decisions on which parts of the business are performed using projects) with business decisions (e.g. the selection of the 'best' projects for the organization), their management (e.g. to maximize the benefits from these projects) and their implementation (e.g. using appropriate ways of managing these projects).

Many of these activities cannot be gleaned from organization charts or role descriptions. However, their existence and smooth integration is crucial for organizations aiming for efficient and economic delivery of their products and/or services to their customers via projects. Organizations establish these networks of project-related activities in order to maximize the internal effectiveness and efficiency of the project-based part of the organization. These networks are often superimposed onto the existing formal structure of an organization, and thus not clearly visible in 
day-to-day work. However, they exist. OPM attempts to conceptualize these networks and their constituting elements (i.e. activities or functions) within the wider scope of permanent organizations that host projects in the form of temporary organizations to deliver beneficial change (Turner and Müller 2003).

This book identifies the elements that make up these networks, and conceptualizes their roles, relationships and institutionalizations within organizations into a cohesive OPM model. This model enables a better understanding of OPM elements and their interactions, popular implementation patterns and their contingencies, as well as providing guidance in designing OPM approaches for organizations.

Historically, OPM is confined to management and governance of three types of activities, namely projects, programmes and portfolios of projects (3Ps). More recently, the academic and practitioner literature has identified a number of activities that are undertaken to make the 3Ps work, such as benefits realization management and organization-wide project management offices (PMOs). However, while these activities are key for successful OPM implementation, the current literature falls short in describing how they link together or are modelled to show their particular roles and the nature of their relationships in the network of OPM activities.

This book addresses this shortcoming by identifying and integrating OPM elements from the recent academic research literature and models their relationships and interactions in a generic way. This is done in the present chapter of the book by providing an introduction to OPM and explaining the logic and method that was used to develop the model. Part 1 provides a detailed description of the model's layers, their elements, the functions of these elements and the ways to measure them. The model's contribution to established organizational theories and the theory of its inner functioning are discussed in Part 2. This part starts with a strategic perspective, by outlining the contribution of the model to resource-based theory. This is followed by an organizational theory perspective, whereby the model's contribution to institutional theory is described. Finally, the model's inner functioning is described using established governmentality theories.

Part 3 addresses the practical use of the model and its different forms of implementation. Two chapters with in-depth case studies provide specific examples of implementations from the private and the public sectors. A third chapter describes the most popular implementation patterns and their context contingencies, based on the results of a cross-sectional study of 20 organizations across different industries, sizes and cultures. Finally, the book ends with a chapter on the design or modification of OPM systems for organizations. 
The book is written for anyone interested in understanding how projects as temporary organizations are embedded and function in their more permanent parent organization. It should be of special interest to practitioners designing the project-based part of an organization, postgraduate students in management and organization studies, early career researchers looking for under-explored areas of research, as well as practising managers in search of understanding how project-related work is integrated in the wider parent organization and how this integration can be optimized for better organizational results.

The rest of this introduction lays the groundwork for a better understanding of the upcoming model by giving a brief look into the development of OPM over time, followed by a description of the model's development.

\section{THE DEVELOPMENT OF OPM}

Crawford (2006) describes the development of OPM as a sequence of two discourses. She states that it initially started with the development and subsequent evolution of tools and techniques of the 3Ps, which then became a distinct body of knowledge for project, programme or portfolio management. This was followed by a turn in focus towards capabilities in the realm of OPM. Jointly, the discussion on tools, techniques and capabilities initiated the second discourse - which addressed the distinction between espoused and practised theories - and led to the development of standards in practising project, programme and portfolio management. Examples include the Project Management Institute's $\left(\mathrm{PMI}^{\circledR}\right)$ practice standards (e.g. PMI 1996). Subsequently, related maturity models for OPM were proposed, turning the discourse towards OPM capability development (e.g. PMI 2003). At that time, the practitioner-oriented literature focused mainly on the existence and expression of functions and processes of the 3Ps in organizations, while the academic literature focused more on the organizational dynamics associated with the emergence of the 3Ps. For example, Aubry, Hobbs and Thuillier (2007, p. 332) defined OPM as 'a new sphere of management where dynamic structures in the firm are articulated as a means to implement corporate objectives through projects in order to maximize value'.

Over time, both the practitioner and academic literature continued to develop within their respective spheres, whereby the former stream recently introduced the concept of principles (i.e. a fundamental truth or proposition that serves as the foundation for a system of belief or behaviour or for a chain of reasoning (Oxford 2019)). This is done to better support processual 
implementations of OPM, whereby processes are understood as sequences of tasks (PMI 2017a). The academic stream of literature took a different turn by emphasizing change and discontinuity in organizations. Here, processes were seen as responses to unpredictable external trajectories requiring a resilient OPM implementation. These processes help in building resilience and the ability to accomplish organizational strategies in a flexible way by reacting to situational contingencies, and then bouncing back to an equilibrium state of stability (Aubry and Lavoie-Tremblay 2018).

Alongside these ontological differences between practitioner and academic literature, a third stream of literature developed, concerned with new insights into OPM. We call these new insights as elements of OPM in the book. These include the different strategies pursued by firms to run the project-based part of their business (Blomquist and Müller 2006), the concept of 'projectification' of organizations and whole societies (Lundin et al. 2015; Midler 1995), and benefits realization management (Chih and Zwikael 2015). While these new perspectives were considered important, the project management community had difficulty positioning them with respect to each other. For example, benefits realization management became simultaneously part of three standards of the same institution - those for governance (PMI 2016), OPM (PMI 2014) and programme management (PMI 2017b). This redundancy is at odds with good governance principles, which call for clearly identifiable accountability of functions (Aras and Crowther 2010; Millstein et al. 1998). Hence, the need for a less redundant and more systematic modelling of OPM arose, to provide academics with an integrated model of often isolated elements, allowing one to further theorize on OPM and its implementation in contextual contingencies, and the relationships of these implementations with organizational results. Simultaneously, the model would guide practitioners through the variety of elements, their functions and interrelationships, in order for them to measure and subsequently profile OPM implementations in organizations. All these could inform the future design of and implementation decisions in OPM for the best interests of the organization.

\section{THE DEVELOPMENT OF THE ‘ONION’ MODEL}

In this section, we briefly describe how the 'onion' model was developed. This development took place from the inside to the outside of the model, starting at the kernel of OPM, that is, with project management, and ending at the boundary of the organization, which is its interface with its market. 
Later in this book we will describe the functions of the elements and layers in more detail, and from the outside of the model to the inside, because that is how an OPM system is expected to be designed and assessed. But first we explain the logic behind the development of the model. The details of the research process are described in Müller, Drouin and Sankaran (2019).

\section{Identification of Elements}

For the development of an integrated model of OPM we had to identify the essential or characteristic measures that are important, or at least frequently practised in OPM. We called these measures as elements. The scope was set to reach from the management of the individual project to the boundaries of its parent organization, that is, the organization's interface with the market. That means that we took an organization-internal perspective toward OPM. An analysis of the mainstream project management research journals (i.e. International Journal of Project Management, Project Management Journal and International Journal of Managing Projects in Business) for OPM-related articles identified the elements listed in Table 1.1. For ease of reading we sort them in accordance with the final model.

The prevalence of the elements naturally differs contingent on the organization and its market. While projects can be assumed to be very prevalent as they can be found in most organizations today, benefits realization management might be much less prevalent as research has shown that only a few organizations practise it. However, research also shows that it is essential for good OPM results. Hence, it is a valid element of the

\section{Table 1.1 Elements derived from the literature}

\begin{tabular}{ll}
\hline Element names & \\
\hline Project-based organization & Portfolio management \\
Project-oriented organization & Portfolio optimization \\
Process-oriented organization & Benefits realization \\
Multi-project strategy/approach & Programme \\
Strategic/organizational PMO & Megaproject \\
Projectification & Project \\
Governance paradigm & Project management methodology \\
Governance model & Policies \\
Governmentality & Relations \\
Governance of project management & Steering group/committee \\
Portfolio strategy & Project management
\end{tabular}


model. At this point, it is important to mention that the list of elements constitutes a snapshot in time, which may exclude items that are identified as essential for good OPM results later or in publications other than those assessed here.

\section{Identification of Layers}

We assessed and classified the OPM elements step by step based on the strength of their logical relationships, which we termed logical cohesion. Those elements with a strong logical cohesion formed a layer of the model. The strength of the logical relationship between the layers was termed logical adhesion, with those layers having the highest levels of logical adhesion being placed adjacent to each other. This resulted in the onion-like shape of the model. For ordering the layers, we selected those elements that have a strong mutual relationship (cohesion) and collectively a strong relationship (adhesion) with the next inner layer. Examples for cohesion and adhesion are provided below. The onion modeling allows the application of the model to various types of organizational structures, such as hierarchies, networks, or hybrids thereof.

The point of departure for model development was the nucleus of OPM activities, that is, the management of the individual project. Hence, project management became the innermost layer.

Project governance layer - The strong logical cohesion between project governance institutions (like steering committees or PMOs), relations (such as legal or psychological contracts), project management methodologies (such as agile or waterfall), and the organization's policies for project management, formed a layer. The cohesion between these elements was considered as higher than their cohesion with other elements. This qualified those elements as part of a layer in the model. Collectively, the elements in this layer had a stronger adhesion to the project management layer than to other layers. In line with the literature, we named this layer project governance and positioned it as the next layer outside the project management layer (Crawford et al. 2008).

Organizational integration layer - The development of the next layer followed the same logic. The particular approach to integrate projectrelated work as either individual projects, programmes of projects or megaprojects results in a strong complementary cohesion. This addresses the form of organizational integration of project-related work, which has a strong influence in the way project governance is performed. Does the organization treat projects as sovereign, autonomous entities with idiosyncratic governance structures (Artto et al. 2008) or as integrative parts of a programme and therefore governed in dependency with other 
projects in a programme (Maylor et al. 2006)? Or does an organization engage in megaprojects, which imposes yet other forms of governance, dealing with a large number of sub-projects and suppliers (Flyvbjerg 2014), potentially creating their own legal entities, such as Special Purpose Vehicles (Sainati, Brookes and Locatelli 2017). Jointly the three elements form a layer which shapes the way project-related work is executed in the organization. We named this layer organizational integration. The characteristics of its three elements influence the choices inside the project governance layer.

Business integration layer - Here, the business opportunities are identified, prioritized and selected, and the management of their benefits initialized. This includes the elements for portfolio strategy, its management and optimization, as well as benefits realization (Killen and Drouin 2017). These elements have a high logical cohesion and collectively they help in making decisions on choices within the organizational integration layer. That is, here is where the decisions are made on which projects, programmes or megaprojects to execute.

OPM governance layer - This layer addresses the governance of groups, or in some organizations the entirety of all (mega)projects/programmes in an organization. This is different from project governance, which addresses the governance of a single project (Müller 2017a). OPM governance determines the organization's governance paradigm for projects (i.e. the ways project managers are controlled within the particular corporate governance settings) and the preferred governance models (such as principlesbased or rule-based models), as well as the governmentality approaches (i.e. the ways in which those in governance roles present themselves to those they govern), plus the governance of project management (i.e. the extent to which project management is developed as a profession and a service within the organization, including the development of project managers and their capabilities). The strong mutual cohesion of these elements makes them part of a layer, which in its entirety strongly influences the choices on the business integration layer.

OPM approach layer - This layer represents upper management's principles for multi-project management, including the decisions on which parts of the organization's business to pursue through projects and, with it, which characteristics of the portfolios to pursue (i.e. the multi-project approach) (Blomquist and Müller 2006), the establishment of strategic or organization-wide project management offices (OPMOs) and their charter, as well as the level of projectification of the organization. The multiproject approach and the OPMO address the overall strategy in terms of handling the entirety of projects in the organization, and projectification determines the extent to which project thinking pervades an organization's 
day-to-day business; for example, in terms of career development ladders for project managers (Midler 1995).

Organizational philosophy layer - This layer groups elements which determine the characteristics of the organization's presence in the marketplace, and its interaction with its partners, suppliers and customers. This is expressed by either being project based (all work is done in projects), project oriented (work is done in projects, even though it could be done in a process) or process oriented (almost all work is done in a production process) (Miterev, Turner and Mancini 2017; Söderlund 2004).

The next step in development was the identification of the nature of the influence that the layers have on each other. For this, we built on Johns' (2006) axiom that behaviour in organizations is context dependent, and identified the enablers, inhibitors or constrains that adjacent layers put on each other. Building further on Johns and the work by Mowday and Sutton (1993), we assumed the predominance of a context-to-element effect, rather than vice versa, and conceptualized the role of each outer layer as the context for the elements in the next inner layer of the onion. This provided the basis for Part 2 of the book, the descriptions of interactions between layers.

The above-mentioned elements, their underlying publications, and the relationships between the elements and layers are further discussed in Parts 1 and 2 of this book. Figure 1.1 shows the final model.

The seven layers span the scope of the entire organization, from the individual project to its governance and structural integration in the organization, its business justification, the organization-wide and strategic governance approaches, up to the presence in the marketplace. More details on the individual elements are provided later in the book. Not all functions of an organization become visible through the above approach, as functions may cover several elements simultaneously. Examples include the human resource management (HRM) function, which is a significant part of projectification, but is also present in governmentality and governance of project management. Another example is information technology (IT), which underlies many of the elements and enables the communication among them. These types of support functions are not addressed in the model. 

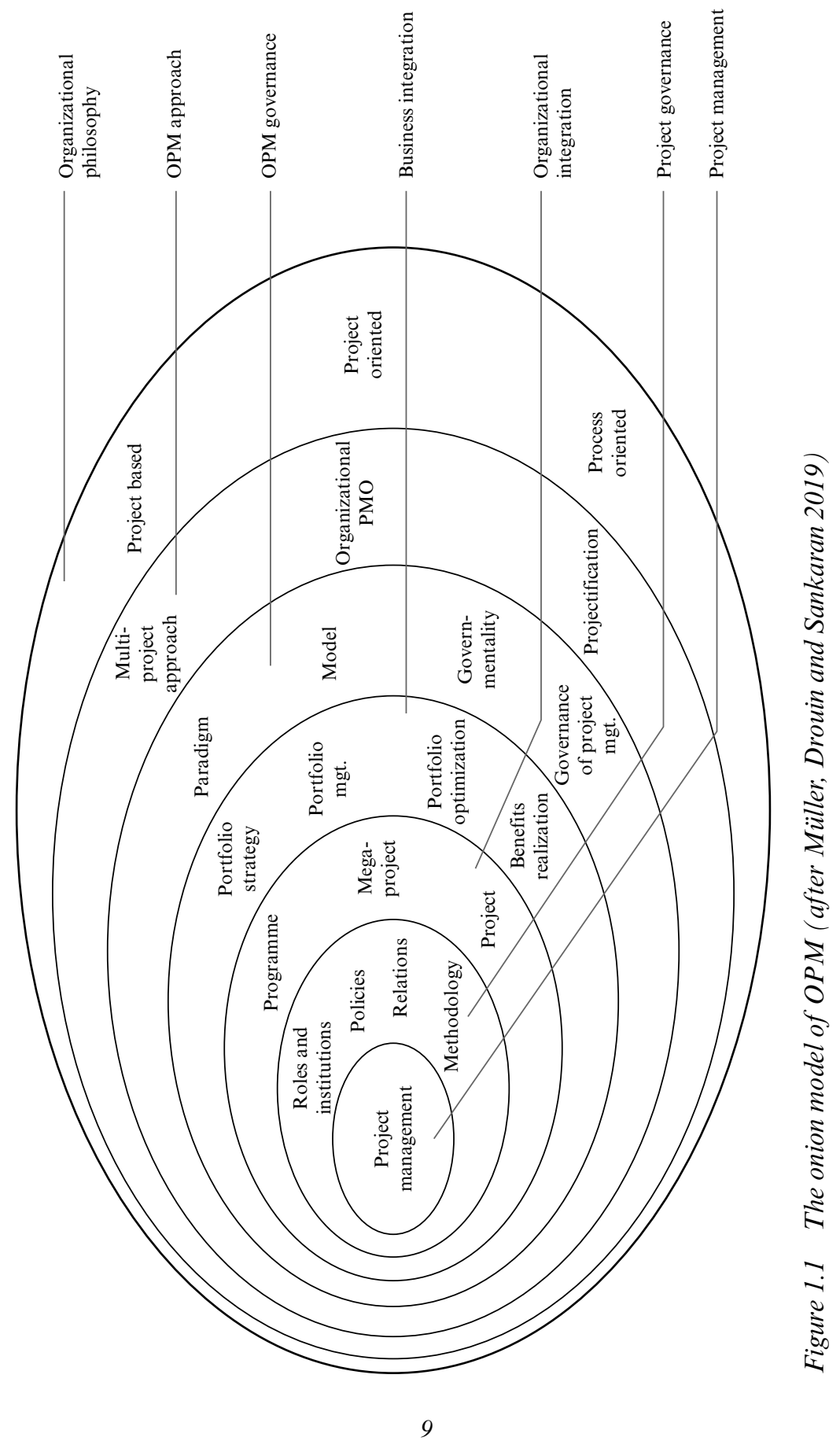